\title{
Minimal Hydrocelectomy with the aid of scrotoscope: a ten-year experience
}

\author{
Yan Bin, Wei Yong-bao, Yin Zhuo,Yang Jin-rui \\ Department of Urology, the Second Xiangya Hospital, Central South University, Changsha, China
}

\section{ABSTRACT}

Background: Since hydrocelectomy remains the choice of surgical treatment of hydrocele and standard surgical procedures may cause postoperative discomfort and complications, a new minimal surgery procedure is needed. The scrotoscope was used for the diagnosis and treatment of intrascrotal lesions. The aim of the study is to illustrate a new minimal hydrocelectomy with the aid of scrotoscope, in an effort to decrease complications.

Materials and Methods: Between 2002 and 2012, 65 patients underwent hydrocelectomy with the aid of a scrotoscope. Before carrying out hydrocelectomy, the scrotoscopy was first used to examine the intrascrotal contents to exclude any pathological lesions. After determining the condition of testis, epididymis and spermatic cord and excluding any other secondary causes of hydrocele, a $2.0 \mathrm{~cm}$ scrotal incision was performed. The parietal tunica vaginalis was then grasped out of scrotum, and the mobilized tunica was excised. The scrotoscopy was then performed again to inspect the intrascrotal contents.

Results: Mean operative time was 35.4 minutes. No major complications occurred during the post-operative follow-up period. Of these 65 patients, 61 underwent scrotoscopy and minimal hydrocelectomy, two patients underwent open hydrocelectomy because thickening of hydrocele wall was identified; two patients with acute inflammation only underwent scrotoscopy. Pathological changes were observed among eight patients. All patients were satisfied with the outcomes.

Conclusions: Minimal hydrocelectomy shows commendable results and fewer complications. The combination of minimal hydrocelectomy and scrotoscopy seems to be an encouraging technique. This novel surgical procedure proves to be a viable option for the diagnosis and treatment of hydrocele.

\section{ARTICLE INFO}

\section{Key words:}

Testicular Hydrocele;

Cystoscopes; Transurethral

Resection of Prostate

Int Braz J Urol. 2014; 40: 384-9

Submitted for publication:

November 21, 2013

Accepted after revision:

March 22, 2014

\section{INTRODUCTION}

Hydrocele refers to a collection of fluid between the parietal and visceral layer of tunica vaginalis (1). Pathogenesis of hydrocele is based on an imbalance between the secretion and reabsorption of this fluid. It is one of the most common benign scrotal pathology that leads patients to visit urologists as outpatients. Acquired hydro- cele affects approximately 1\% of men and most of them are more than 40 years old (2).

Acquired hydrocele can occur secondary to intrascrotal infection, regional or systemic diseases, inguinal or scrotal surgery or neoplasm, but most hydroceles are commonly idiopathic in origin (3). To diagnose hydrocele, clinical examination and ultrasound are the first options. However, in certain situations, a definitive diagnosis 
is not possible on the basis of the clinical and ultrasound findings. Muglia et al. found ultrasound to be inconclusive in $5.02 \%$ of their patients with scrotal disease (4). Endoscopy is an instrument used to detect and visualize the intrascrotal contents directly. Gerris $\mathrm{J}$ et al. first reported the use of scrotal endoscopy (5). Shafik et al. presented some further experience with scrotoscope in the diagnosis and treatment of intrascrotal lesions (6). Our previous study showed that scrotoscope was far more precise than B-ultrasonography in diagnosing intrascrotal lesions (7).

The conventional treatment of a symptomatic hydrocele is surgical and hydrocelectomy remains the most common method of treatment. Standard surgery brings about postoperative discomfort, a temporary limitation of normal activity and complications including prolonged pain, recurrence, hematoma, infection and injury to the scrotal contents (8). Various minimal invasive procedures including minimal access hydrocelectomy, fenestration, aspiration and sclerotherapy were described $(9,10)$. However, all the minimal procedures were performed without the thorough observation of intrascrotal contents. Therefore, the surgery could be performed under the condition that other underlying intrascrotal pathology is missed, for preoperative clinical examination or ultrasound could misdiagnose these conditions.

We innovatively combined the scrotoscope and a minimal access hydrocelectomy for the first time. To our knowledge there are very few published reports on scrotoscope for intrascrotal diseases used as an operative instrument. This novel technique appeared to be feasible, effective, well tolerated and safe for acquired hydrocele.

\section{MATERIALS AND METHODS}

\section{Patients}

We obtained approval for this study from the Institutional Review Board of The Second Xiangya Hospital, Central South University. Informed consent was obtained from all patients in the study. The informed consent was written and specified in the operative consent. All the participants were informed of the risks and benefits and completed the informed consent process.
Between April 2002 and December 2012, a total of 65 patients, aged 19-67 years (mean age 50.8 years) presenting with non-septated hydroceles underwent our novel surgical treatment. Before undergoing the surgery, all patients were evaluated with history and clinical examination followed by scrotal ultrasound to determine the nature of the hydrocele and to rule out any other intrascrotal pathological conditions including testicular malignancy, infection and hernia. Patients who were diagnosed as non-septated hydrocele were included in this study. Exclusion criteria included patients with a multiseptated hydrocele, communicating hydrocele or other scrotal abnormalities, such as tumor, infection, spermatocele or hernia.

\section{Surgical technique}

The patients received general anesthesia, spinal anesthesia, or caudal anesthesia. With the patients in lithotomy position, the scrotal skin was thoroughly examined and sterilized with povidone-iodine. A small scrotal incision of about $1.0 \mathrm{~cm}$ was performed. Bluntly dissection was then performed through the scrotal layer until the tunica sac was disclosed (Figure-1). Generally, the hydrocele fluid would flow out and a $50 \mathrm{~mL}$ syringe was used to aspirate the fluid. The amount and appearance of the fluid were recorded and all of it was sent for bacterial culture to rule out any potential infection. Typically, the fluid was clear yellow in appearance and negative result in bacterial culture.

As no specialized scrotocrope exists, we used cystoscope or resectoscope as scrotoscope. The sheath of scrotoscope with the obturator was passed through the incision and tunica sac (Figure-2). Keeping the drip fusion of isotonic solution inflowing, the scrotum was maintained appropriate distended. The tunica sac wall including parietal and visceral tunica was checked thoroughly while the instrument was advanced. The testis, epididymis and spermatic cord were then examined from the anterior, posterior and both lateral aspects to find out any potential pathology. Biopsy of the suspicious lesions was performed when intrascrotal abnormalities were identified. 
Figure 1 - A $1.0 \mathrm{~cm}$ incision is made in the middle to lower part of the scrotum.

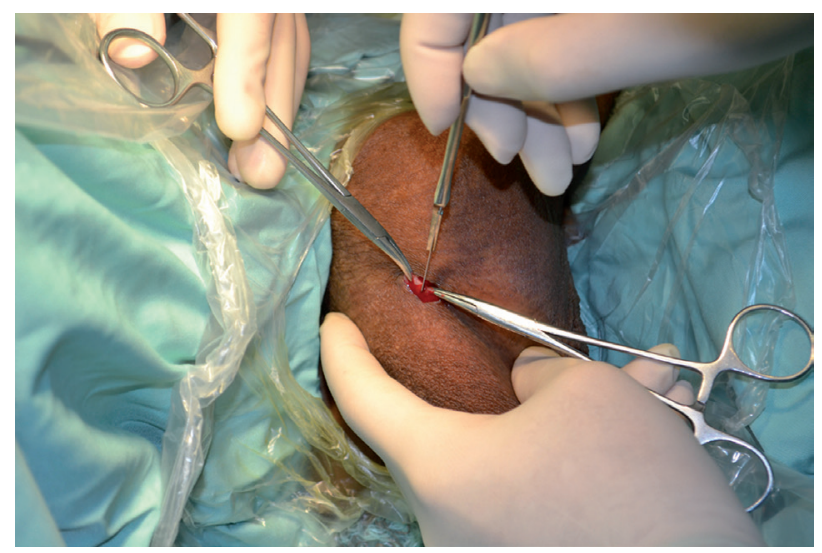

The sheath was then removed and the incision was elongated to $2.0 \mathrm{~cm}$ using a scalpel knife. With the aid of the index finger, the parietal tunica vaginalis was grasped and bluntly dissected from adherent tissues. Using Allis forceps, the sac was then gently pulled out of the scrotal incision (Figure-3). The mobilized tunica vaginalis was excised by electrocautery. Active bleeding was clamped and ligated. The remaining sac was

Figure 2 - A resectoscoope is used as scrotocrope and is placed into tunica sac through the $1.0 \mathrm{~cm}$ incision.

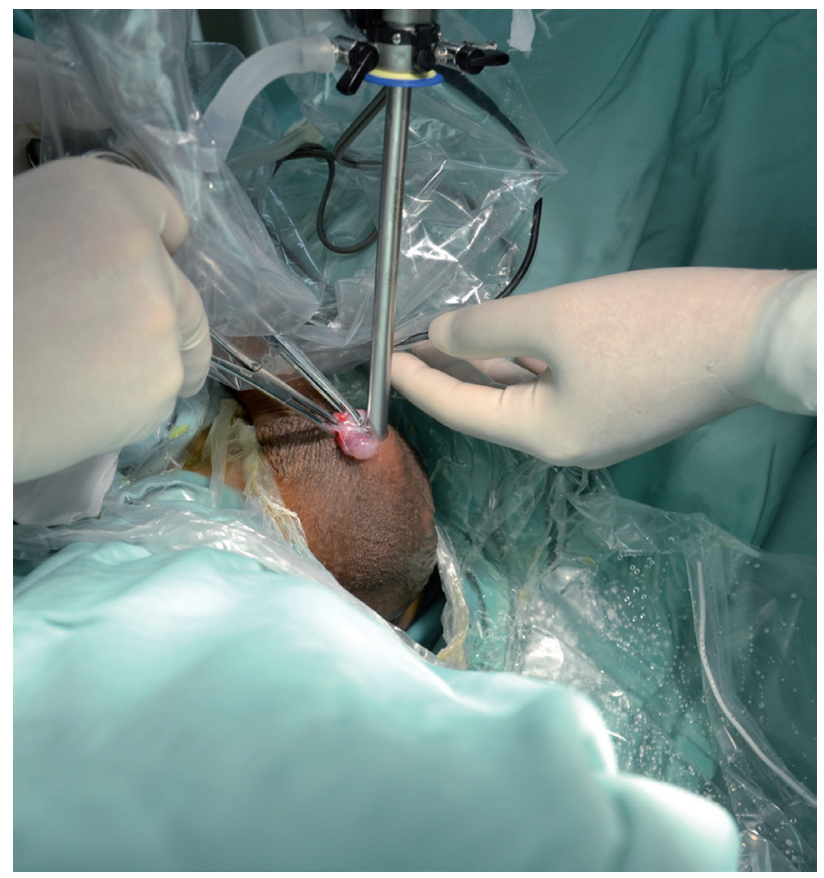

Figure 3 - Tunica vaginalis is grasped, bluntly dissected and gently pulled out of the scrotal incision.

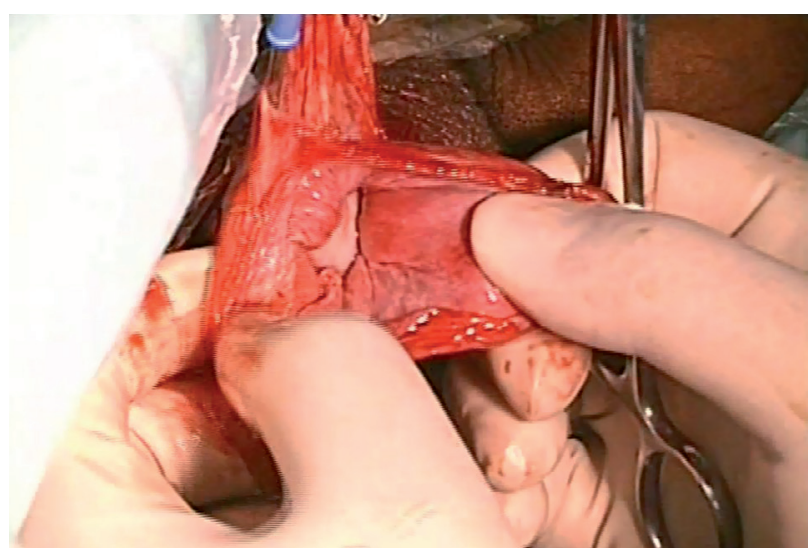

replaced back in the scrotum. All excised tissue specimens were sent for pathological examination to rule out any epididymal or vasal structures in the specimen.

The scrotoscopy was performed again to reexamine the intrascrotal contents to exclude any active bleeding or neglected lesion. The incision was then closed with an absorbable suture with placement of a rubber strip drain. All patients had the drains removed 24 hours after surgery. A wound dressing was applied with the scrotum elevated for 2-3 days. Antibiotics were administrated as anti-infective prophylactic therapy. Additional course of antibiotics were given depending on intrascrotal condition and the results of bacterial culture.

All patients were followed up in the outpatient office 4 weeks after the procedure. Subsequent follow-up was made in the outpatient or with a telephone call. All patients were followed at 6 months intervals for a mean of 26 months (range 12-30 months). The outcome criteria included: pain was assessed using visual analogue scale (VAS) of 10 ('0' meaning no pain at all and '10' meaning worst possible pain), and patient scoring 3 were considered to have pain; infection was defined as the presence of either positive microbial culture from wound discharge, or a combination of fever, pain, local erythema and discharge at the incision site. A patient was considered satisfied if the following criteria were met: decrease in pain; decrease in size of hydrocele; relief of any hydrocele-related disability; and satisfaction with overall 
experience and results. Success was defined as no perceptible fluid, improvement in symptoms of pain or discomfort and patient satisfaction at the last follow-up. Criteria for failure were recurrence of the hydrocele and no improvement in symptoms including pain and discomfort.

\section{RESULTS}

A total of 65 patients aged 19-67 years old (mean age 50.8 years) from April 2002 to December 2012 were admitted to our hospital. Mean operative time was 35.4 minutes (range 25.4 to 38 minutes). Overall mean follow-up was 16 months (range 12 to 22 months). Mean volume of fluid aspirated during the procedure was $85 \mathrm{~mL}$ (range 22 to $285 \mathrm{~mL}$ ). The color of aspirated fluid of 61 patients (61/65) was clear yellow, of two patients was turbid yellow and of 2 two patients was light red. All the bacterial cultures of fluids were negative. All the pathological examination of the resected tissue proved to be normal tunica.

Of these 65 patients, 61 underwent scrotoscopy and minimal hydrocelectomy. Two patients underwent open hydrocelectomy following scrotosctopy because thickening of hydrocele wall was identified through scrotoscope and minimal hydrocelectomy was not suitable. Two patients only underwent scrotoscopy because acute inflammation was found through scrotoscope (Figure-4). Abnormalities were identified in eight patients of 65 and biopsies were then performed. These eight patients included two thickening of hydrocele wall, two acute inflammation and four chronic inflammation. Patients were able to resume usual daily activities an average of 4 days (range 2 to 7) after surgery.

The main complications included mild to moderate scrotal edema in four patients and two with moderate amount of postoperative pain. These two complications mostly resolved within 1-2 days without the need for pharmacologic treatment or surgical, endoscopic, and radiologic interventions. No other complications occurred. Complications of all patients were included into grade I in terms of Clavien-Dindo classification system. Intrascrotal acute inflammation was found through scrotoscope in these two patients and the hydroceletomy was not performed and the pain subsided five days later after surgery. No patients experienced postoperative hematoma or wound infection. Postoperative recurrence was not seen in any of these patients. All patients were satisfied with this procedure. The procedure was thought to be successful for all the patients (100\%).

\section{DISCUSSION}

An acquired hydrocele is one of the most common benign scrotal pathological changes which affects approximately 1\% of men and is mostly seen after age 40 years. Most acquired hydroceles are idiopathic in origin, but some may result from a reaction to tumors, infection or trauma. Pathogenesis of hydrocele is based on an imbalance between the secretion and reabsorption of the fluid (11). The standard hydroceletomy is a common surgical procedure, though various methods of treatment for acquired hydrocele were described, such as hydrocele aspiration and sclerotherapy, endoscopic hydrocele ablation $(12,13)$. Hydroceletomy has advantages over these treatments in terms of the recurrence rate and patient satisfaction. Hydrocelectomy remains as the gold standard

Figure 4 - While the drip fusion was suspended, acute inflammation of parietal tunica was demonstrated.

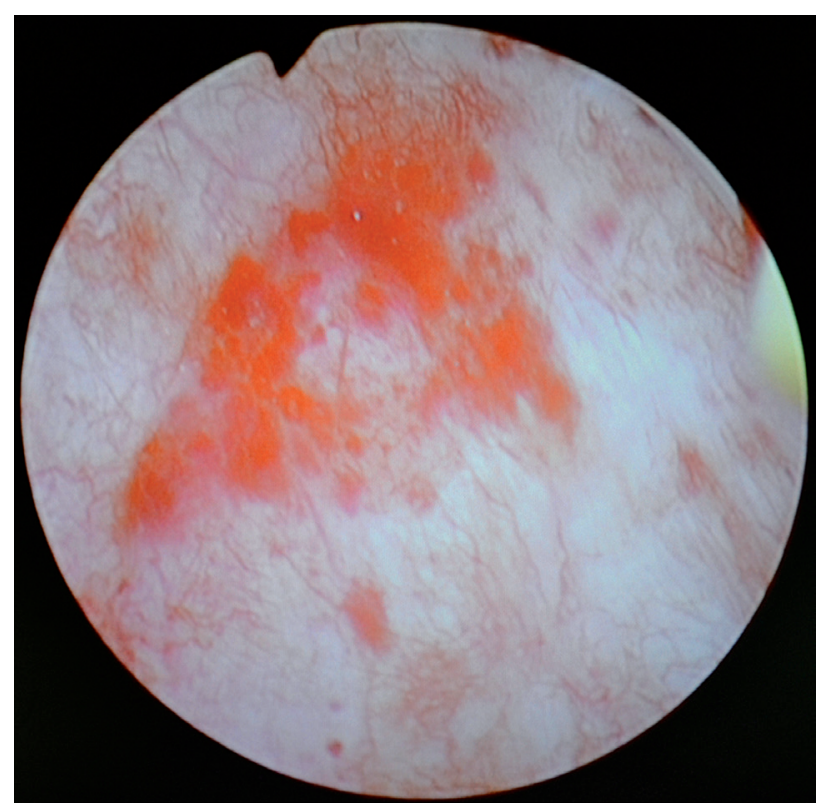


modality for the treatment of hydrocele (14). But it has the disadvantages of discomfort and complications including mild to moderate incidence rate of recurrences, hematomas and infections; none of which had happened in our new surgery $(8,15)$. New minimal hydrocelectomy are designed to overcome these disadvantages.

Some new minimal hydrocelectomy procedures were reported to excise hydrocele sacs through small incisions. The procedures showed minimal complications, decreased discomfort, and without recurrence. $(9,10)$. They proved to be a viable and promising option for the surgical management of idiopathic hydrocele. But these procedures were performed without inspection of intrascrotal contents. Some pathological lesions resulting in hydroceles like infection, trauma or aseptic inflammation may be overlooked even when surgery was done. Though preoperative clinical examination and ultrasound can diagnose most of the intrascrotal lesions correctly, some pathological changes may still be missed.

Scrotal endoscopy was first reported in 1988 (5). It was used in the examination of intrascrotal contents and taking biopsies of pathological lesions (6). We started to utilize scrotoscopic technique to perform direct observation and biopsies of scrotal lesions and removal of lumps in 1990. We made a comparative study of the diagnostic preciseness of scrotoscope and B-ultrasonography on scrotal lesions. It showed that scrotoscope is far more precise than B-ultrasonography in the total diagnostic effectiveness (73.3\% / 46.7\%) and distinguishing a benign lump from a tumor (87.6\% / 60\%) (7).

In a study of endoscopic hydrocele ablation, electrocautery or laser were used to ablate tunica vaginalis endoscopically. In comparison with open hydrocelectomy, this approach appeared to be effective and well-tolerated with minimal postoperative discomfort. However, some drawbacks of this approach including need of extra trocar, risk of injuring the testis and fulguration of the testis or epididymis may limit its wide use in clinical practice (12). We started to utilize scrotoscope to treat hydrocele in 2002. Scrotoscope was used to inspect intrascrotal contents to rule out any pathological changes which could be missed by clinical examination and ultrasound. Minimal hydrocelectomy was then performed followed by second time scrotoscopy.
Mean operative time in our study was 35.4 minutes (range 25.4 to 40 minutes) which was longer than other minimally invasive procedures but was shorter than endoscopic hydrocele ablation and open hydrocelectomy $(9,10,12)$. It was reasonable since extra scrotoscopy was performed. All patients were considered to be satisfied with their treatment. The most common complications were mild to moderate scrotal edema (4/65) and scrotal pain (2/65). Scrotal hematoma, severe scrotal edema, wound infection or recurrence was not seen in all cases. These values are comparable to other similar reports.

One advantage of this surgery procedure is the combination of scrotoscope with minimal hydrocelectomy. Because of the scrotoscopy performed, intrascrotal abnormalities which may be misdiagnosed by clinical examination and ultrasound could be detected. This study has showed that pathological changes have been identified in eight patients. The hydroceletomy was avoided in two patients with acute inflammation and open hydroceletomy was performed in two patients with thickening of tunica wall. These pathological changes would not be observed if minimal hydrocelectomy was completed alone.

Scrotoscope enables the surgeon to pick the most suitable surgery procedure for these patients. Comparing with standard hydrocelectomy, another advantage of this procedure is that the testis and spermatic cord are not handled and not removed out of the scrotum. Thus, there is fewer chance that spermatic cord or testicular torsion happen during and after operation. However, this procedure is not suitable for patients with septated hydroceles. For these patients, scrotoscopy is not recommended and open hydrocelectomy is necessary. One limitation of this study was that it was a retrospective and nonrandomized study in nature. To provide more accurate and convincing information, a prospective randomized trial is highly recommended and it is the next study we are working on.

\section{CONCLUSIONS}

The scrotoscope proves to be a safe and effective diagnostic and therapeutic technique. Our findings demonstrate that minimal hydrocelectomy with the aid of a scrotoscope has the advantages 
of minimal incision, decreased complications and low rate of recurrence while clearly detecting intrascrotal contents.

\section{ACKNOWLEDGEMENTS}

The study was supported by the Fundamental Research Funds for the Central Universities of Central South University in 2013 (2013zzts095).

\section{CONFLICT OF INTEREST}

None declared.

\section{REFERENCES}

1. Kapur P, Caty MG, Glick PL: Pediatric hernias and hydroceles. Pediatr Clin North Am. 1998; 45: 773-89.

2. Leung ML, Gooding GA, Williams RD: High-resolution sonography of scrotal contents in asymptomatic subjects. AJR Am J Roentgenol. 1984; 143: 161-4.

3. Rubenstein RA, Dogra VS, Seftel AD, Resnick MI: Benign intrascrotal lesions. J Urol. 2004; 171: 1765-72.

4. Muglia V, Tucci S Jr, Elias J Jr, Trad CS, Bilbey J, Cooperberg PL: Magnetic resonance imaging of scrotal diseases: when it makes the difference. Urology. 2002; 59: 419-23.

5. Gerris J, Van Camp C, Van Neuten J, Gentens P, Van Camp K: Scrotal endoscopy in male infertility. Lancet. 1988; 1: 1102.

6. Shafik $A$ : The scrotoscope. A new instrument for examining the scrotal contents. Br J Urol. 1990; 65: 209-10.

7. Yang J, Huang $\mathrm{X}$ : Comparative study of the diagnostic preciseness of scrotoscope and B-ultrasonography on scrotal lesions. Zhonghua Wai Ke Za Zhi. 1996; 34: 173-5.

8. Swartz MA, Morgan TM, Krieger JN: Complications of scrotal surgery for benign conditions. Urology. 2007; 69: 616-9.

9. Saber A: New minimally access hydrocelectomy. Urology. 2011; 77: 487-90.

10. Onol SY, Ilbey YO, Onol FF, Ozbek E, Arslan B, Akba A: A novel pull-through technique for the surgical management of idiopathic hydrocele. J Urol. 2009; 181: 1201-5.

11. Woodward PJ, Schwab CM, Sesterhenn IA: From the archives of the AFIP: extratesticular scrotal masses: radiologicpathologic correlation. Radiographics. 2003; 23: 215-40.

12. Ho GT, Ball RA, Schuessler W, Kavoussi LR: Efficacy of endoscopic hydrocele ablation. J Endourol. 1993; 7: 71-4.

13. Francis JJ, Levine LA: Aspiration and sclerotherapy: a nonsurgical treatment option for hydroceles. J Urol. 2013; 189: 1725-9.

14. Khaniya S, Agrawal CS, Koirala R, Regmi R, Adhikary S: Comparison of aspiration-sclerotherapy with hydrocelectomy in the management of hydrocele: a prospective randomized study. Int J Surg. 2009; 7: 392-5.

15. Kiddoo DA, Wollin TA, Mador DR: A population based assessment of complications following outpatient hydrocelectomy and spermatocelectomy. J Urol. 2004; 171: 746-8.

\footnotetext{
Correspondence address: Jin-rui Yang, $\mathrm{PhD}$ Department of Urology, The Second Xiangya Hospital, Central South University, Changsha, 410011, China E-mail: 2xiangya2010@gmail.com
} 